\title{
Sex ratio of the mutation frequencies in haemophilia A: coagulation assays and RFLP analysis
}

\author{
A H J T Bröcker-Vriends, F R Rosendaal, J C van Houwelingen, E Bakker, \\ G J B van Ommen, J J P van de Kamp, E Briët
}

\begin{abstract}
Coagulation and RFLP data from 41 families with an isolated haemophilia $A$ patient were used to estimate the sex ratio of mutation frequencies $(v / \mu)$. Based on the results of coagulation assays in all the female relatives investigated, $v / \mu$ was estimated to be $12 \cdot 1$ by the maximum likelihood method $(95 \%$ confidence interval 3.8 to 62.5 ). In order to avoid the possible influence of germline mosaicism, an additional analysis was performed in which only the results in the mothers and grandmothers of an isolated patient were included. The $v / \mu$ ratio was then estimated to be $5 \cdot 2(95 \%$ confidence interval $1 \cdot 8$ to $15 \cdot 1$ ).

Because an estimate of $v / \mu$ based on all available RFLP data can easily be biased in favour of males, we set up a model in which only information on the grandparental derivation of the patient's $X$ chromosome was used, irrespective of the generation in which
\end{abstract}

Clinical Genetics Centre, University Hospital Leiden (33), Rijnsburgerweg 10, 2333 AA Leiden, The Netherlands.

A H J T Bröcker-Vriends, J J P van de Kamp

Department of Clinical Epidemiology, University Hospital, Leiden, The Netherlands.

F R Rosendaal

Department of Medical Statistics, State University, Leiden, The Netherlands.

$\mathrm{J} C$ van Houwelingen

Department of Human Genetics, State University, Leiden, The Netherlands.

E Bakker, G J B van Ommen

Department of Haematology, University Hospital, Leiden, The Netherlands.

E Briët

Correspondence to Dr Bröcker-Vriends.

Received for publication 27 July 1990.

Revised version accepted for publication 15 March 1991. the mutation actually occurred. In this way $v / \mu$ was estimated to be minimally 4 .

The probability of carriership for mothers of an isolated haemophilia $A$ patient amounts to $86 \%$ with a sex ratio of $5 \cdot 2$. Although this would imply that $14 \%$ of the mothers are not carriers of the disease in the classical sense, they may be mosaic for the mutation and, therefore, also at risk of transmitting the mutation more than once.

One-third of patients with haemophilia have no affected male relatives. ${ }^{12}$ Therefore, the question of which of the female relatives of these isolated patients are at risk for being a carrier is of major importance in genetic counselling for haemophilia.

The probability of carriership for female relatives of isolated patients is determined by the origin of the mutation in the family and depends on the ratio of the mutation frequency in males $(v)$ and females $(\mu)$. If mutations occur predominantly in males, a high proportion of the mothers of an isolated patient will be carriers of the disease. On the other hand, the more often mutations occur in females, the more isolated patients will be sons of non-carrier mothers and be so-called 'true sporadic patients'.

The sex ratio of the mutation frequencies $(v / \mu)$ for haemophilia has been estimated by different methods. In 1935, Haldane ${ }^{3}$ showed that for $\mathrm{X}$ linked recessive diseases $\nu / \mu$ can be derived from the proportion of sporadic patients of all patients $(x)$ by the equation $x=\mu /(2 \mu+v)$, provided that genetic equilibrium is present. This proportion $x$ is expected to be $1 / 3$, when $v / \mu$ equals 1 . The proportion of sporadic cases is expected to be lower than $1 / 3$ when mutations occur more frequently in males than in females, and also when affected males have significant reproductive fitness. Theoretically, the fitness of carrier females may be of influence as well. A modification of Haldane's equation, which accounts for all these factors, was derived by Holloway and Smith. ${ }^{4}$ The proportion of sporadic patients $(\mathbf{x})$ can be determined either by segregation analysis or by carrier detection tests in mothers of an affected male. 
In addition to genetic equilibrium, Haldane's method requires complete or unbiased ascertainment of affected males or mothers of an affected male. If ascertainment is incomplete, families with more than one affected male are more likely to be included, which leads to an underestimation of the proportion of sporadic patients and, consequently, to an overestimation of $v / \mu$. In order to eliminate ascertainment bias, Davie and Emery ${ }^{5}$ and, later, Winter ${ }^{6}$ developed a method by which the proportion of sporadic patients $(x)$ is estimated using only data from families with an isolated patient.

In haemophilia, genetic equilibrium is highly unlikely. Life expectancy of patients with haemophilia has markedly increased over the years, ${ }^{78}$ and so has their reproductive fitness. ${ }^{9}$ Therefore, the fitness of the patients who have contributed to the population under study is difficult to assess, and the assumption of a steady state is unwarranted.

Recently, we have argued that, under certain conditions, limitation of pedigree analysis to families with an (initially) isolated haemophilia patient allows an estimate of $v / \mu$ independent of the fitness of affected males and of the existence of genetic equilibrium. ${ }^{10}$

During the last five years, developments in DNA technology have provided new means to determine the origin of mutations. Restriction fragment length polymorphisms (RFLPs) within or closely linked to the factor VIII gene can be used to trace the inheritance of the patient's $\mathrm{X}$ chromosome. The generation in which the mutation has occurred may thus be defined, as well as the male or female origin of the mutation. Müller and Grimm ${ }^{11}$ proposed a method to estimate $\nu / \mu$ from the proportion of patients of all patients who inherited their maternal grandfather's $\mathrm{X}$ chromosome. As in the method of Haldane, this estimate is very sensitive to ascertainment bias, in such a way that overrepresentation of familial cases may cause an underestimate of $v / \mu .^{1213} \mathrm{~A}$ method theoretically free of ascertainment bias was developed by Karel et al..$^{14}$ This method, however, requires so many pedigrees of a specific, infrequently occurring type that it is only feasible through large scale collaboration over a long period.

We have obtained an estimate of $v / \mu$ from the results of coagulation assays and RFLP analysis in 41 families with an isolated haemophilia A patient. For the interpretation of the coagulation assays, we have developed a method that resembles the method described by Winter. ${ }^{6}$ From the RFLP data, information on the grandparental origin of the patient's X chromosome was used to estimate $v / \mu$. For this purpose the model of Müller and Grimm ${ }^{11}$ was modified for application in families with an isolated haemophilia A patient.
Subjects and methods

\section{SUBJECTS}

We investigated subjects from 41 families with an isolated haemophilia A patient. All the families were referred to our centre during the years 1984 to 1989 for genetic counselling of the female relatives.

Extensive family histories, which covered information about the great grandfathers, grandfathers, brothers, cousins, nephews, uncles, and great uncles of the patient, were taken to exclude previously affected males in the pedigree. Since the occurrence of mild haemophilia cannot be reliably assessed in this way, we restricted the analysis to families in which the patient had a moderately severe $(n=6)$ or a severe $(n=35)$ form of haemophilia $A$, that is, less than $5 \%$ clotting factor VIII activity.

\section{COAGULATION ASSAYS}

Coagulation assays were performed in all the mothers of the isolated patients, in 19 of the 41 maternal grandmothers, and in 66 other female relatives, which included sisters, aunts, cousins, and nieces.

For this purpose, $9 \mathrm{ml}$ of blood was obtained and added to $1 \mathrm{ml}$ of sodium citrate (3.2\%). Immediately after collection, the citrated blood was spun at high speed and the platelet-poor plasma was dispensed in capped disposable plastic tubes. The plasma was then deep frozen and stored at $-70^{\circ} \mathrm{C}$. The tubes were thawed immediately before testing and assayed within two months of blood collection. We took care that the sampling, processing, and storage of blood was accomplished by a minimum number of experienced persons and in a consistent way on all occasions.

Factor VIII coagulant activity (VIII:c) was measured by a one stage clotting assay, ${ }^{15}$ and von Willebrand factor antigen (vWf:Ag) by Laurell immunoelectrophoresis. ${ }^{16}$ Bleeding time (method of Ivy) and haematocrit were checked, and blood groups were determined for statistical purposes.

\section{RFLP ANALYSIS}

Restriction fragment length polymorphism (RFLP) analysis was carried out in all of the 41 families. In 13 families both maternal grandparents could be investigated, in eight families only the maternal grandmother, and in the remaining 20 families neither of them was available for DNA analysis. However, in some families the RFLP haplotype of the $\mathrm{X}$ chromosomes of the grandparents could be deduced from those of their children.

We used two intragenic RFLPs, F8a/BclI ${ }^{17}$ and $\mathrm{F} 8 \mathrm{~b} / \mathrm{XbaI},{ }^{18}$ and two extragenic RFLPs, St14/ 
Taq $\mathrm{I}^{19}$ and DX13/BglII. ${ }^{20}$ The recombination frequency between the extragenic markers and the clotting factor VIII gene is approximately $5 \% .^{21}$

The methods of DNA analysis used in our centre have been described previously for each of the aforementioned RFLPs. ${ }^{223}$

\section{STATISTICAL METHODS}

For each woman, the likelihood ratio of carrier/noncarrier based on the results of coagulation assays (LR-VIII) was calculated by discriminant analysis with correction for age and blood group. ${ }^{24}$ LR-VIIIs greater than 100:1 or smaller than $1: 100$ were trimmed to $100: 1$ or $1: 100$, respectively, to avoid a disproportionate influence of outlying values in further statistical procedures. The LR-VIIIs for the females were combined with the pedigree information, more specifically the number and genetic status of the males, to estimate the sex ratio of mutation frequencies by the maximum likelihood method. The results of RFLP analysis were evaluated separately. The proportion of mothers of an isolated patient who are expected to be carriers of the disease was calculated according to Rosendaal et al. ${ }^{10}$

Estimation of the sex ratio of mutation frequencies by pedigree analysis and coagulation assays

We have derived an expression for the likelihood of the outcome of carrier detection tests on women in a specific pedigree, conditional on the number and genetic status of the male relatives. In this expression the likelihood is a function of two parameters designated $\delta_{1}$ and $\delta_{2}$. The parameter $\delta_{1}$ equals $\Omega / \mu$, where $\Omega$ is the probability of carriership for the woman in the pedigree, for whom there is no reliable previous information, the progenitor. The parameter $\delta_{2}$ equals $(\mu+v) / \mu$. The derivation of the expression is given in the appendix.

From the likelihood for the outcome of carrier detection tests in all the pedigrees investigated, which is the product of the individual likelihoods (see appendix), the parameters $\delta_{1}$ and $\delta_{2}$ can be estimated by the maximum likelihood method. Confidence intervals can be obtained by calculation of the standard error by the second derivative of the log likelihood function or by taking all values of the unknown parameter for which log likelihood is equal to or greater than the maximum log likelihood minus $1.92(1.92=0.5 \times 3.84 ; 3.84$ is the 95 th centile of the $\chi_{1}^{2}$ distribution). Because in this study the log likelihoods for $v / \mu\left(v / \mu=\delta_{2}-1\right)$ appeared to be asymmetrical about their mode, we have chosen the last approach to obtain the confidence interval for $v / \mu$. The log likelihood curves for $v / \mu$ were constructed by maximisation of the original log likelihood with respect to $\delta_{1}$ (at a fixed value of $v / \mu$ ).
In this study, it was impossible to estimate $\delta_{1}$ with a high degree of precision. This is owing to lack of substantial information, that is, carrier detection tests, in the more remote generations. We found, however, that the log likelihood curve for $v / \mu$ was not notably influenced by entering different values for $\delta_{1}$.

Estimation of the sex ratio of mutation frequencies by RFLP analysis

From the results of RFLP analysis, the grandparental derivation of the $\mathrm{X}$ chromosome of an affected male can be deduced, irrespective of the generation in which the mutation occurred. We have evaluated the significance of the grandparental derivation of an isolated patient's $\mathrm{X}$ chromosome for the sex ratio of mutation frequencies $(v / \mu)$.

The grandpaternal $\mathrm{X}$ chromosome (GP-X) may appear in an affected grandson because of a mutation during spermatogenesis resulting in a carrier daughter, who subsequently transmitted this $\mathrm{X}$ chromosome to her son $(v / 2)$ or because of a mutation in the patient's mother $(\mu)$. The grandmaternal $\mathrm{X}$ chromosome (GM-X) may appear in an affected grandson because of carriership of the maternal grandmother $(1 / 4 \Omega)$, by a new mutation in the grandmother resulting in a carrier daughter who subsequently transmitted the $\mathrm{X}$ chromosome to her son $(\mu / 2)$, or because of a mutation in the patient's mother $(\mu)$. If we consider only families with no affected male relatives in the grandparental or more distant generations, the prior probability of carriership for the grandmother $(\Omega)$ approximates $2 \mu+2 v \cdot{ }^{10}$ The conditional probability for the patient's $X$ chromosome having been inherited either from the grandfather or the grandmother is then

GP-X: $v / 2+\mu$

GM-X: $(2 \mu+2 v) / 4+\mu / 2+\mu$

and the ratio for the grandparental derivation becomes

$$
\text { GP-X/GM-X }=(v+2 \mu) /(v+4 \mu)=(k+2) /(k+4)
$$

wherein $v / \mu$ is denoted by $k$. It can be seen that for this type of family, the ratio GP-X/GM-X is never expected to exceed 1 , even if mutations occurred exclusively in males.

We restricted our investigation to families with an isolated patient which means that we excluded those in which the grandmothers had more than one descendant with haemophilia. The prior probability of carriership for the grandmothers in our study population is thus a fraction ( $t$ ) of the prior probability for all the grandmothers with no previous 
family history of haemophilia. Accordingly, the ratio GP-X/GM-X in families with an isolated patient equals

$\mathrm{GP}-\mathrm{X} / \mathrm{GM}-\mathrm{X}=(\mathrm{k}+2) / \mathrm{t}(\mathrm{k}+1)+3$

and depends not only on $v / \mu$, but also on the fraction of carrier grandmothers included in the analysis. If none of the carrier grandmothers is included $(t=0)$, the ratio GP-X/GM-X is expected to equal 1 in case $\mu=\nu$ and to exceed 1 in case $\nu>\mu$. The more carrier grandmothers included ( $t>0)$, the less likely that a higher mutation frequency in males will result in predominant inheritance of the grandpaternal $X$ chromosome (fig 1). In other words, whatever the value of $t$, when the patient's $X$ chromosome is found to be derived more often from the grandfather than from the grandmother, this finding always indicates a higher mutation frequency in males. A minimum value of $v / \mu$ can be derived from the ratio GP-X/GM-X, assuming $\mathrm{t}=0$.

\section{Results}

ESTIMATION OF THE SEX RATIO OF MUTATION FREQUENCIES BY PEDIGREE ANALYSIS AND COAGULATION ASSAYS

First, a maximum likelihood estimate of $v / \mu$ was obtained by use of the information from the pedigrees and the LR-VIII for all the female relatives investigated. The mutation ratio $(v / \mu)$ was estimated

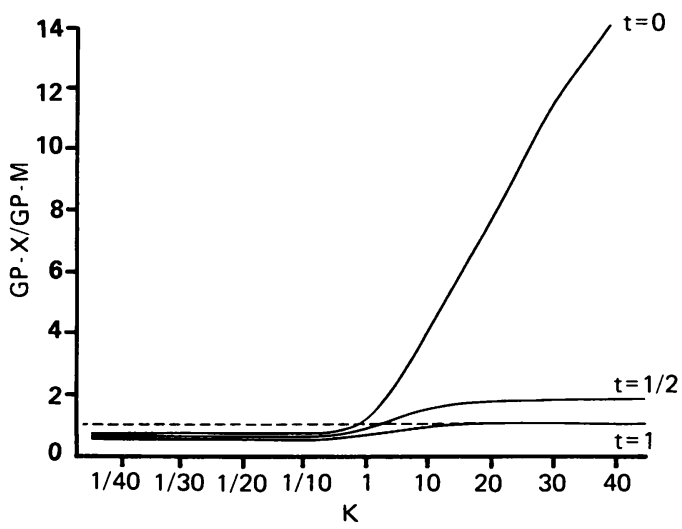

Figure 1 Theoretical relationship between the grandparental derivation of a patient's $X$ chromosome and $v / \mu$. The relationship applies to families in which no male relatives are affected in the grandparental or more remote generations of the index case and is expressed by the equation $G P-X / G M-X=(k+2) / t(k+1)+3$, where ' $t$ ' is the fraction of carrier grandmothers included in the study. $G P-X=$ grandpaternally derived $X$ chromosome.

$G M-X=$ grandmaternally derived $X$ chromosome. $k=v / \mu$.

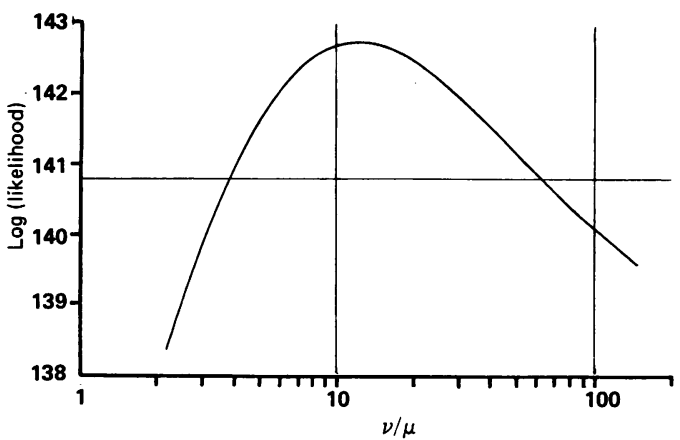

Figure 2 Log likelihood curve for the sex ratio of mutation frequencies $(\nu / \mu)$ based on the results of coagulation assays in all the female relatives of isolated haemophilia $A$ patients investigated $(n=41)$. The horizontal line corresponds to the maximum log likelihood minus 1.92 and has been used to compute the $95 \%$ confidence interval of $v / \mu$ (see Methods).

to be $12 \cdot 1$ with a $95 \%$ confidence interval of 3.8 to $62 \cdot 5$ (fig 2).

In four of the families the patient's mother was more likely to be a carrier than a non-carrier, not on the basis of her own factor VIII levels but on the basis of those of her daughter. In these cases the daughters conferred on their mother an LR which almost equalled that of a second son with haemophilia (table 1). Although normal factor VIII values may have been found in the mother because of preferential lyonisation of the mutated $\mathrm{X}$ chromosome, germline mosaicism may be an explanation as well.

In order to avoid the possible influence of this phenomenon, we performed an additional analysis, in which only the results of coagulation assays in mothers and grandmothers of the isolated patients were included. The ratio $v / \mu$ was then estimated to

Table 1 Contradictory results of coagulation assays between the mother of an isolated patient and her daughter.

\begin{tabular}{lc}
\hline Pedigree and subject & LR-VIII* \\
\hline A19† & 0.08 \\
Mother & $>100$ \\
Daughter & 0.03 \\
A29 & $>100$ \\
$\quad$ Mother & 0.3 \\
Daughter & $>100$ \\
A30 & \\
Mother & 0.02 \\
Daughter & $>100$ \\
Mother & \\
Daughter & \\
\hline
\end{tabular}

* Likelihood ratio carrier/non-carrier based on the results of factor VIII and von Willebrand factor assays according to Green $e t a l^{4}$; for further statistical procedures values above 100:1 were trimmed to $100: 1$.

t Somatic and germline mosaicism for a partial deletion in the FVIII gene was found in the mother. ${ }^{25}$ 


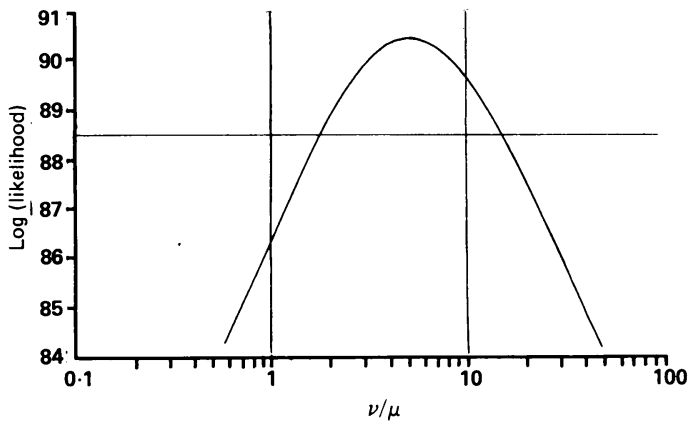

Figure 3 Log likelihood curve for the sex ratio of mutation frequencies $(v / \mu)$ based on the results of coagulation assays in the mothers and grandmothers of isolated haemophilia $A$ patients $(n=41)$. The horizontal line corresponds to the maximum log likelihood minus 1.92 and has been used to compute the $95 \%$ confidence interval of $v / \mu$ (see Methods).

be $5 \cdot 2$ with a $95 \%$ confidence interval of $1 \cdot 8$ to $15 \cdot 1$ (fig 3).

ESTIMATION OF THE SEX RATIO OF MUTATION FREQUENCIES BY RFLP ANALYSIS

From the results of RFLP analysis the grandparental derivation of the patient's $\mathrm{X}$ chromosome could be deduced in 22 of the 41 families (table 2). The X chromosome of the patient was found to be derived from the grandfather twice as often as from the grandmother (GP-X/GM-X=2). From fig 1 it follows that this finding corresponds with a minimum value for $v / \mu$ of 4 .

\section{COMBINED RESULTS OF RFLP ANALYSIS AND COAGULATION ASSAYS}

The most probable level of mutation for the grandpaternally and grandmaternally derived $\mathrm{X}$ chromosomes is depicted in fig $\mathbf{4}$ and is based on the results of coagulation assays in the mother and grandmother of the patient. Fourteen of the 15 mothers in whom the at risk $\mathrm{X}$ chromosome was of paternal origin had an LR-VIII above 1, 13 even above 10, so that in these cases the mutation most probably occurred in the grandfather of the patient. However,

Table 2 Grandparental derivation of the $X$ chromosome of isolated haemophilia $A$ patients by RFLP analysis $(n=41)$.

\begin{tabular}{lccc}
\hline & \multicolumn{3}{c}{ RFLP } \\
\cline { 2 - 4 } Grandparent & Intragenic & Extragenic & Total \\
\hline Grandfather & 9 & 6 & 15 \\
Grandmother & 2 & 5 & 7 \\
Unknown & - & - & 19 \\
\hline
\end{tabular}

five of the seven mothers in whom the at risk $\mathrm{X}$ chromosome was of maternal origin had an LR-VIII below 1, so that in these cases the mutation most probably occurred in the mother of the patient. In the remaining two cases the mother as well as the grandmother of the patient had an LR-VIII above 1, which indicates that the mutation had occurred in the great grandparental or an even more remote generation.

Fourteen of the 22 mothers included in this figure are thus most probably carriers (LR-VIII $>1$ ) by new mutation. It is striking that, in all these women, the mutation originated in the paternal $\mathrm{X}$ chromosome.

\section{PROBABILITY OF CARRIERSHIP FOR MOTHERS OF AN} ISOLATED PATIENT

The theoretical probability of carriership for mothers of an isolated patient was calculated to be $93 \%$ for a mutation ratio of $12 \cdot 1$ and $86 \%$ for a mutation ratio of $5 \cdot 2$. In addition to carriers in the classical sense, the first percentage may include women who are mosaic for the mutation, whereas the second percentage does not. In both analyses the remaining percentages of mothers, $7 \%$ and $14 \%$

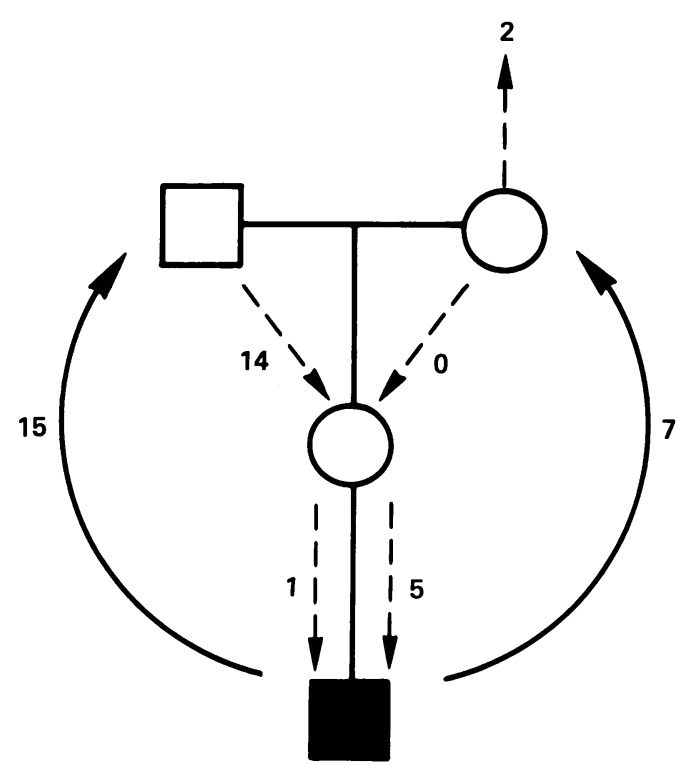

Figure 4 Most probable level of mutation for the grandpaternally and grandmaternally derived $X$ chromosomes based on the results of coagulation assays in the mother and grandmother of the patient. Solid arrows, grandparental derivation by RFLP analysis; broken arrows, probable level of mutation by RFLP analysis and coagulation assays ( $L R-V I I I>1$ carrier, $L R-V I I I<1$ non-carrier). 
respectively, are expected to be either non-carrier or mosaic for the mutation.

\section{Discussion}

In this study, we have found evidence that haemophilia A mutations occur more frequently in males than in females. Based on pedigree analysis and coagulation assays in 41 families with an isolated patient, the sex ratio of mutation frequencies $(v / \mu)$ was conservatively estimated to be $5 \cdot 2$ with a $95 \%$ confidence interval of 1.8 to $15 \cdot 1$. The results of RFLP analysis were in agreement with this estimate.

Most of the earlier studies indicated a higher mutation frequency in males as well, ${ }^{1026-31}$ though some did not. ${ }^{32-35}$ The studies based on segregation analysis ${ }^{1026273235}$ have been criticised for ascertainment bias and the techniques used to correct for this. Rosendaal $e t a l^{10}$ virtually eliminated the possibility of selective ascertainment by using only information from pedigrees with an initially isolated patient. In this study, a slight preponderance of the male mutation frequency, in a ratio of $2: 1$, was found. In addition to poor ascertainment, many of the studies based on carrier detection tests ${ }^{262-313334}$ have been hampered by unreliable carrier detection methods or an inadequate interpretation of them. Winter $e^{t} a l^{31}$ strictly controlled carrier detection tests and their interpretation according to the World Health Organization recommendation. ${ }^{36}$ Based on the results of coagulation assays in 21 families with an isolated patient, the sex ratio $(v / \mu)$ was estimated to be 9.6 with a $95 \%$ confidence interval of $2 \cdot 2$ to $41 \cdot 5$. Recently, a meta-analysis of six of these earlier studies, in which an estimate of $v / \mu$ was obtained, confirmed a higher mutation frequency in males $(v / \mu: 3 \cdot 1) .^{10}$

The method which we have developed according to the model of Winter ${ }^{6}$ allows, in principle, inclusion of all types of pedigrees, irrespective of the number of affected males and of the generation(s) in which they occur. By basing the likelihood for the outcome of carrier detection tests on the pedigree information, that is, the number and genetic status of the male relatives, selection for a specific type of pedigree does not affect the estimated value of the parameters. At present, in view of the possible occurrence of mosaicism for mutations, we consider it advisable to limit this type of analysis to pedigrees with an isolated patient. Alternatively, when a certain value for $v / \mu$ has been assumed, the method can be used for individual probability calculations in genetic counselling.

In this study, the estimate of $v / \mu$ appeared to be virtually independent of the prior probability of carriership for the woman in the pedigree designated the progenitor. In most of our pedigrees it concerned the great grandmother of the patient. The probability of carriership for the progenitor is determined by the mutation frequency in males and females as well as by the reproductive fitness in affected males. When more generations occur between patient and progenitor, the impact of $\Omega$ on the carrier status of the mother of an isolated patient and, hence, the sex ratio of mutation frequencies, becomes less. As a consequence, the reproductive fitness of affected males, whatever the value, cannot be of major influence either.

When families with an isolated patient are ascertained through female relatives of the patient, the estimate of $v / \mu$ may be flawed by the referral pattern of these women. Since we are one of the major centres for carrier detection of haemophilia in the Netherlands, we cannot exclude the possibility that relatively more complex cases, that is, women who are difficult to classify, have been referred to us. In that case our estimate of $v / \mu$ would be too low.

We have estimated $v / \mu$ based exclusively on the results of coagulation assays in mothers and grandmothers of an isolated patient to avoid the possible influence of germline mosaicism. There appeared to be a considerable difference in the estimate based on coagulation assays in all available female relatives, $12 \cdot 1$ versus $5 \cdot 2$. The actual finding of germline mosaicism in one of the families included in this study ${ }^{25}$ has proven that the estimate of $12 \cdot 1$ may indeed be too high because of this phenomenon. The impact of germline mosaicism in haemophilia is, as yet, unknown. Therefore, a more conservative approach to estimate $v / \mu$ may be warranted.

To date, information from DNA studies on the origin of mutations is limited. Based on the results of RFLP analysis and of coagulation assays in families with an isolated patient, Bernardi $e t a^{37}$ arrived at a higher mutation frequency in males. It should be noted, however, that the chance to define the origin of the mutation by RFLP analysis depends on the information provided by the family and is easily biased in favour of males. We have chosen an approach by which limited, though unbiased, information from the RFLP studies, namely the grandparental derivation of the patient's $\mathrm{X}$ chromosome, was used to estimate $v / \mu$. For that purpose, we have defined the relationship between $v / \mu$ and the ratio of grandpaternally and grandmaternally derived $\mathrm{X}$ chromosomes specifically for families with an isolated patient. A minimum estimate of $v / \mu$ could thus be obtained. In addition, we have shown that predominant inheritance of the grandmaternal $\mathrm{X}$ chromosome does not exclude a higher mutation frequency in males.

When the actual gene defect is known, the origin of the mutation can be unequivocally shown. In 22 haemophilia A families, the origin of the mutation has been reported..$^{38-48}$ Although the figures are small, they favour a higher mutation frequency in 
males. In contrast, for another $\mathrm{X}$ linked recessive disease, Duchenne muscular dystrophy (DMD), segregation analysis and carrier detection tests as well as recent molecular analysis have not provided convincing evidence for a difference in mutation frequency between males and females. ${ }^{490} \mathrm{DMD}$ is caused by a deletion in the dystrophin gene in 60 to $65 \%$ of the cases, ${ }^{51} 52$ whereas this applies to only 4 to $6 \%$ of the haemophilia A cases. ${ }^{45} 53$ These figures might indicate that there is a correlation between the type of mutation and its male or female origin. The findings for haemophilia A show that deletions might be more frequently associated with somatic or germline mosaicism than point mutations, which in turn might be related to the possible mechanisms of mutagenesis causing the respective mutations. Recently, recurrence risks owing to mosaicism have been reported for DMD. ${ }^{50}$ In view of a possible relation between the type of mutation and the occurrence of mosaicism, caution is needed in applying these figures to haemophilia $\mathbf{A}$ families as well.

Knowledge of the sex ratio of mutation frequencies is important for genetic counselling in families with an isolated haemophilia A patient. The higher the $v / \mu$, the higher is the probability of carriership for mothers of an isolated patient. The most conservative estimate of $v / \mu$ in the present study $(5 \cdot 2)$ results in a probability of carriership of $86 \%$. Although this implies that $14 \%$ of the mothers are not expected to be carriers in the classical sense, these mothers are not exempted from transmission of the same mutation to other children because of the possibility of germline mosaicism.

As early as 1935 , Haldane ${ }^{3}$ not only postulated a sex difference in mutation rates, but also hinted at the possibility of mosaicism. The occurrence of somatic and germline mosaicism has now been definitely established by molecular analysis. The prevalence of this phenomenon and related recurrence risks need to be evaluated further. In addition, studies at the gene level may provide a definite answer on a possible sex difference of mutation frequencies, not only for all gene defects combined, but also for the different types of mutation separately. International collaboration will be essential to achieve this goal.

Finally, in view of current knowledge one cannot but wonder: who is a 'truly sporadic case'?

We wish to thank J C F M Dreesen and C van Alebeek for the molecular analysis of the families; R J S Ysseldijk, C J M van Dijk-Kempen, M J Kret, H M E Roosen-van Dokkum, and E Noorlander for performing the coagulation assays; $\mathbf{R} M$ ClaassenTegelaar for the organisation of the family studies; and Drs K E Davies, J L Mandel, and R M Lawn, who kindly provided us with the DX13, St14, and factor VIII probes, respectively. Mrs F L Brussen-
Walta diligently prepared the manuscript. This work was financially supported by the Praeventie Fonds, Grant 28-1244.

\section{Note}

Pedigree and laboratory data are available upon request from the authors or the editorial office.

1 Barrai I, Cann HM, Cavalli-Sforza LL, De Nicola P. The effect of parental age on rates of mutation for hemophilia and evidence for differing mutation rates for hemophilia $A$ and $B$. Am $\mathcal{F}$ Hum Genet 1968;20:175-96.

2 Rosendaal FR, Smit C, Varekamp I, et al. Verslag van een in 1985 gehouden landelijk onderzoek onder mensen met hemofilie. In: Hemofilie in Nederland 3. Amsterdam: Dutch Society of Haemophilia Patients (NUHP), 1987.

3 Haldane JBC. The rate of spontaneous mutation of a human gene. f Genet 1935;31:317-26.

4 Holloway SM, Smith C. Equilibrium frequencies in X-linked recessive disease. Am f Hum Genet 1973;25:388-96.

5 Davie AM, Emery AEH. Estimation of proportion of new mutants among cases of Duchenne muscular dystrophy. $\mathcal{F}$ Med Genet 1978;15:339-45.

6 Winter RM. Estimation of male to female ratio of mutation rates from carrier-detection tests in X-linked disorders. $A m \mathcal{F}$ Hum Genet 1980;32:582-8.

7 Larsson SA. Life expectancy of Swedish haemophiliacs, 18311980. Br f Haematol 1985;59:593-602.

8 Rosendaal FR, Varekamp I, Smit C, et al. Mortality and causes of death in Dutch haemophiliacs, 1973-1986. Br $\mathcal{F}$ Haematol 1989;71:71-6.

9 Rosendaal FR, Smith C, Varekamp I, et al. Modern haemophilia treatment: medical improyements and quality of life. F Intern Med 1990;228:633-40.

10 Rosendaal FR, Bröcker-Vriends AHJT, Van Houwelingen JC, et al. Sex ratio of the mutation frequencies in haemophilia $A$ : estimation and meta-analysis. Hum Genet 1990;86:139-46.

11 Müller CR, Grimm T. Estimation of the male to female ratio of mutation rates from the segregation of $\mathrm{X}$-chromosomal DNA haplotypes in Duchenne muscular dystrophy families. Hum Genet 1986;74:181-3.

12 Müller CR, Meng G, Grimm T. DNA-marker typing may help to estimate the male to female ratio of the mutation rates in DMD. Proceedings 7th International Congress of Human Genetics, Berlin, 1986.

13 Te Meerman GJ, Karel ER, Ten Kate LP. Ascertainment bias and power of procedures to estimate differences between male and female mutation rates. Hum Genet 1987;75:296.

14 Karel ER, Te Meerman GJ, Ten Kate LP. On the power to detect differences between male and female mutation rates for Duchenne muscular dystrophy, using classical segregation analysis and restriction fragment length polymorphisms. $A m$ f Hum Genet 1986;38:827-40.

15 Veltkamp JJ, Drion EF, Loeliger EA. Detection of the carrier state in hereditary coagulation disorders I. Thromb Haemost 1968;19:279-303.

16 Laurell CB. Quantitative estimation of protein by electrophoresis in agarose gels containing antibodies. Anal Biochem 1966;15:45-52.

17 Gitschier J, Drayna D, Tuddenham EGD, White RL, Lawn RM. Genetic mapping and diagnosis of haemophilia $A$ achieved through a BclI polymorphism in the factor VIII gene. Nature 1985;314:738-40.

18 Wion KL, Tuddenham EGD, Lawn RM. A new polymorphism in the factor VIII gene for prenatal diagnosis in hemophilia A. Nucleic Acids Res 1986;11:4534-42.

19 Oberlé I, Camerino G, Heilig R, et al. Genetic screening for hemophilia A (classic hemophilia) with a polymorphic DNA probe. N Engl f Med 1985;312:682-6.

20 Harper K, Pembrey ME, Davies KE, Winter RM, Hartley D, Tuddenham EGD. A clinically useful DNA probe closely linked to haemophilia A. Lancet 1984;ii:6-8.

21 Peake I, Lillicrap DP, Liddel MB, Matthews RJ, Bloom AL. Linked and intragenic probes for haemophilia A. Lancet 1985;ii: $1003-4$. 
22 Bröcker-Vriends AHJT, Briët E, Quadt R, et al. Genotype assignment of haemophilia $A$ by use of intragenic and extragenic restriction fragment length polymorphisms. Thromb Haemost 1987;57:131-6.

23 Bröcker-Vriends AHJT, Briët E, Kanhai HHH, et al. First trimester prenatal diagnosis of haemophilia A: two years' experience. Prenat Diagn 1988;8:411-21.

24 Green PP, Mannucci PM, Briët E, et al. Carrier detection in hemophilia A: a cooperative international study. II Th efficacy of a universal discriminant. Blood 1986;67:1560-7.

25 Bröcker-Vriends AHJT, Briët E, Dreesen JCFM, et al. Somatic origin of inherited haemophilia A. Hum Genet 1990;85:28892.

26 Haldane JBS. The mutation rate of the gene for haemophilia and its segregation in males and females. Ann Eugen 1947;13:262-71.

27 Hermann J. Der einfluss des zeugungsalters auf die mutationen zu Hämophilie A. Hum Genet 1966;3:1-16.

28 Biggs R, Rizza CR. The sporadic case of haemophilia A. Lancet 1976;ii:431-3.

29 Hathaway HS, Lubs ML, Kimberling WJ, Hathaway WE. Carrier detection in classical hemophilia. Pediatrics 1976;57:251-4.

30 Ratnoff OD, Jones PK. The laboratory diagnosis of the carrier state for classic hemophilia. Ann Intern Med 1977;86:521-8.

31 Winter RM, Tuddenham EGD, Goldman E, Matthews KB. A maximum likelihood estimate of the sex ratio of mutation rates in haemophilia A. Hum Genet 1983;64:156-9.

32 Kosower N, Christiansen R, Morton NE. Sporadic cases of hemophilia and the question of a possible sex difference in mutation rates. Am f Hum Genet 1962;14:159-69.

33 Ekert H. Carrier studies in 'simplex families'. Thromb Haemost 1977;38:721-3.

34 Ananthakrishnan R, D'Souza S. Some aspects of the occurrence of new mutations in haemophilia. Hum Hered 1979;29:90-4.

35 Barrai I, Cann HM, Cavalli-Sforza L L, Barbujani G, De Nicola P. Segregation analysis of hemophilia A and B. Am f Hum Genet 1985;37:680-99.

36 Akhmeteli MA, Aledort LN, Alexiantes S. Methods for the detection of haemophilia carriers: a memorandum. Bull WHO 1977;55:675-702.

37 Bernardi F, Marchetti G, Bertagnolo V, et al. RFLP analysis in families with sporadic hemophilia A. Hum Genet 1987;76:253-6.

38 Gitschier J, Wood WI, Tuddenham EGD, et al. Detection and sequence of mutations in the factor VIII gene of haemophiliacs. Nature 1985;315:427-30.

39 Youssoufian $\mathrm{H}$, Kazazian $\mathrm{HH}$ Jr, Phillips DG, et al. Recurrent mutations in haemophilia $\mathrm{A}$ give evidence for $\mathrm{CpG}$ hotspots. Nature 1986;324:380-2.

Appendix Estimation of the sex ratio of mutation frequencies from carrier detection tests in an $X$ linked recessive disorder.

The subjects $\left(D_{n}\right)$ in each pedigree $(S)$ are consecutively denoted $D_{1}$ to $D_{n v}$ for the females and $D_{n v+1}$ to $D_{n}$ for the males. $D_{1}$ is the woman in the pedigree for whom there is no previous information, the progenitor. The mother of the respective subjects is denoted $M_{i}(i=2, \ldots, n)$. Let $D_{i}$ be 1 if a subject, male or female, carries the abnormal gene and let $D_{i}$ be 0 if a subject does not carry the abnormal gene. The probability for each subject in a pedigree to carry the abnormal gene, given the genetic status of the mother, is:
40 Youssoufian H, Antonarakis SE, Aronis S, Tsiftis G, Phillips DG, Kazazian HH Jr. Characterisation of five partial deletions of the factor VIII gene. Proc Natl Acad Sci USA 1987;84:3772-6.

41 Youssoufian H, Wong C, Aronis S, Platokoukis H, Kazazian HH Jr, Antonarakis SE. Moderately severe hemophilia A resulting from Glu $\rightarrow$ Gly subsitution in exon 7 of the factor VIII gene. Am $\mathcal{F}$ Hum Genet 1988;42:867-71.

42 Youssoufian H, Antonarakis SE, Bell W, Griffin AM, Kazazian HH Jr. Nonsense and missense mutations in hemophilia $A$ estimate of the relative mutation rate at CG dinucleotides. Am f Hum Genet 1988;42:718-25.

43 Higuchi M, Kochhan L, Olek K. A somatic mosaic for haemophilia A detected at the DNA level. Mol Biol Med 1988;5:237.

44 Gitschier J. Maternal duplication associated with gene deletion in sporadic hemophilia. Am f Hum Genet 1988;43:274-9.

45 Higuchi M, Kochhan L, Schwaab R, et al. Molecular defects in hemophilia $A$ : identification and characterization of mutations in the factor VIII gene and family analysis. Blood 1989;3:1045-51.

46 Gitschier J, Levinson B, Lehesjoki AE, de la Chapelle A. Mosaicism and sporadic haemophilia: implications for carrier determination. Lancet 1989;i:273-4.

47 Wehnert M, Herrmann FH, Wulff K. Partial deletions of factor VIII gene as molecular diagnostic markers in haemophilia $\mathrm{A}$. Dis Markers 1989;7:113-17.

48 Levinson B, Lehesjoki AE, de la Chapelle A, Gitschier J. Molecular analysis of hemophilia A mutations in the Finnish population. Am ₹ Hum Genet 1990;46:53-62.

49 Moser $\mathrm{H}$. Review of studies on the proportion and origin of new mutants in Duchenne muscular dystrophy. In: Ten Kate LP, Pearson PL, Stadhouders AM, eds. Research into the origin and treatment of muscular dystrophy. Amsterdam: Excerpta Medica, 1984:41-52.

50 Bakker E, Veenema H, Den Dunnen JT, et al. Germinal mosaicism increases the recurrence risk for 'new' Duchenne muscular dystrophy mutations. F Med Genet 1989;26:553-9.

51 Koenig M, Beggs AH, Moyer M, et al. The molecular basis for Duchenne versus Becker muscular dystrophy: correlation of severity with type of deletion. Am f Hum Genet 1989, 45:498-506.

52 Den Dunnen JT, Grootscholten PM, Bakker E, et al. Topography of the Duchenne muscular dystrophy (DMD) gene: FIGE and CDNA analysis of 194 cases reveals 115 deletions and 13 duplications. Am f Hum Genet 1989;45:835-47.

53 Kazazian HH Jr, Wong C, Youssoufian H, Scott AF, Phillips DG, Antonarakis SE. Haemophilia A resulting from de novo insertion of $\mathrm{L} 1$ sequences represents a novel mechanism for mutation in man. Nature 1988;332:164-6.

For the progenitor:

$\mathrm{P}\left(\mathrm{D}_{1}=1\right)=\Omega$

$\Theta_{1}$

For females by new mutation:

$\mathrm{P}\left(\mathrm{D}_{\mathrm{i}}=1 \mid \mathrm{D}_{\mathrm{mi}}=0\right)=\mu+v \quad \Theta_{2} \quad \mathrm{i}=2, \ldots, \mathrm{n} v$

For males by new mutation:

$\mathrm{P}\left(\mathrm{D}_{\mathrm{i}}=1 \mid \mathrm{D}_{\mathrm{mi}}=0\right)=\mu \quad \Theta_{3} \quad \mathrm{i}=\mathrm{n} v+1, \ldots, \mathrm{n}$

For males and females by inheritance:

$P\left(D_{i}=1 \mid D_{m i}=1\right)=P\left(D_{i}=0 \mid D_{m i}=1\right)=1 / 2$

$$
\mathrm{i}=2, \ldots, \mathrm{n}
$$

The probability for the genetic status of the subjects belonging to a specific pedigree is then the product of the individual probabilities 


$$
\begin{aligned}
\mathbf{P}\left(\mathrm{D}_{1}, \ldots, \mathrm{D}_{\mathrm{n}}\right) & =\mathbf{P}\left(\mathrm{D}_{1}\right) \prod_{2}^{\mathrm{n}}\left(\mathrm{D}_{\mathrm{i}} \mid \mathbf{D}_{\mathrm{mi}}\right) \\
& =\Theta_{1}^{\mathrm{D} 1} \Theta_{2}^{\mathrm{F}} \Theta_{3}^{\mathrm{H}}\left(\frac{1}{2}\right)^{\mathrm{c}}
\end{aligned}
$$

where $F$ is the number of new female mutants, $H$ is the number of new male mutants, and $C$ is the number of children of carrier mothers. It should be noted that this expression includes pedigrees in which no mutation occurred $\left(\mathrm{D}_{1}+\mathrm{F}+\mathrm{H}=0\right)$ as well as pedigrees in which a mutation occurred more than once $\left(D_{1}+F+H>1\right)$.

If we restrict the possible type of pedigrees to pedigrees with only one mutational event $\left(D_{1}+F+H=1\right)$, the probability for the subjects of a specific pedigree becomes

$$
\mathrm{P}\left(\mathrm{D}_{1}, \ldots, \mathrm{n}\right)=\left(\mathrm{D}_{1} \Theta_{1}+\mathrm{F \Theta}_{2}+\mathrm{H} \Theta_{3}\right)\left(\frac{1}{2}\right)^{\mathrm{C}} .
$$

Since the selection of the investigated pedigrees from this population of pedigrees is unclear and depends on the genetic status of the males, we have used the probability of carriership for the females conditional on the genetic status of the males. Accordingly, the probability for the genetic status of the females in a specific pedigree is given by the expression

$$
\begin{aligned}
P\left(D_{1}, \ldots, D_{n v} \mid D_{n v+1}, \ldots, D_{n}\right)= & \frac{\left(D_{1} \Theta_{1}+F \Theta_{2}+H \Theta_{3}\right)\left(\frac{1}{2}\right)^{C}}{\sum\left(D_{1} \Theta_{1}+F \Theta_{2}+H \Theta_{3}\right)\left(\frac{1}{2}\right)^{C}} \\
& D_{1}, \ldots, D_{n v} \\
& D_{1}+F+H=1
\end{aligned}
$$

and depends only on the ratio of $\Theta_{1}, \Theta_{2}$, and $\Theta_{3}$. If one sets $\Theta_{3}$ at 1 , the probability depends on two parameters $\Theta_{1} / \Theta_{3}=\delta_{1}$ and $\Theta_{2} / \Theta_{3}=\delta_{2}$, where $\delta_{1}$ equals $\Omega / \mu$ and $\delta_{2}$ equals $(\mu+v) / \mu$. The $\delta$ dependence of the probability for the genetic status of the females in a specific pedigree can be denoted by

$P_{\delta}\left(D_{1}, \ldots, D_{n v} \mid D_{n v+1}, \ldots, D_{n}\right)$.

Let $\mathrm{LR}-\mathrm{VIII}_{\mathrm{i}}$ be the likelihood ratio carrier/noncarrier based on the results of factor VIII assays for the female subjects $(i=1, \ldots, n v)$. The probability for the genetic status of the females together with the results of factor VIII assays, conditional on the number and genetic status of the males, is then proportional to

$P_{\delta}\left(D_{1}, \ldots, D_{n v} \mid D_{n v+1}, \ldots, D_{n}\right) \prod_{1}^{n v} L R-V I I I_{i}^{D_{i}}$

Hence, the likelihood for the results of factor VIII assays in the females of a specific pedigree is given by

$$
L(\delta)=\sum_{\substack{D_{1}, \ldots, D_{n v} \\ D_{1}+F+H=1}} P_{n v}\left(D_{n}, \ldots, D_{n v+1} \mid D_{n}, \ldots, D_{n}\right) \prod_{1}^{n v} L R-V I I I_{i}^{D_{i}}
$$

The likelihood for the results of factor VIII assays in all the investigated pedigrees is expressed by

$$
L(\delta)=\prod_{s}\left\{\begin{array}{l}
\Sigma P_{\delta}\left(D_{1}, \ldots, D_{n v} \mid D_{n v+1}, \ldots, D_{n}\right) \prod_{1}^{n v} L R-V I I I I_{i}^{D_{i}} . \\
D_{1}, \ldots, D_{n v} \\
D_{1}+F+H=1
\end{array}\right.
$$

